\title{
Pseudomonas aeruginosa eradication therapy and risk of acquiring Aspergillus in young children with cystic fibrosis
}

\author{
Sabariah Noor Harun, ${ }^{1,2}$ Nicholas H G Holford, ${ }^{3}$ Keith Grimwood, ${ }^{4}$ \\ Claire E Wainwright, ${ }^{5,6}$ Stefanie Hennig, ${ }^{2}$ On behalf of Australasian Cystic Fibrosis \\ Bronchoalveolar Lavage (ACFBAL) study group
}

- Additional material is published online only. To view please visit the journal online (http://dx.doi.org/10.1136/ thoraxjn-2018-211548).

${ }^{1}$ School of Pharmaceutical Sciences, Universiti Sains Malaysia, USM, , Malaysia ${ }^{2} \mathrm{~S} c h$ ool of Pharmacy, The University of Queensland, Brisbane, Queensland, Australia ${ }^{3}$ Department of Pharmacology and Clinical Pharmacology, University of Auckland,

Auckland, New Zealand ${ }^{4}$ Menzies Health Institute Queensland, Griffith University and Gold Coast University Hospital, Gold Coast, Queensland, Australia

${ }^{5}$ Respiratory and Sleep Medicine, Lady Cilento Children's Hospital, South Brisbane, Queensland, Australia

${ }^{6}$ Child Health Research Centre The University of Queensland, Brisbane, Queensland, Australia

\section{Correspondence to} Dr Stefanie Hennig, School of Pharmacy, The University of Queensland, Brisbane, QLD 4072, Australia;

s.hennig@uq.edu.au

Received 17 January 2018 Revised 31 March 2019

Accepted 8 May 2019

Published Online First

15 June 2019

Check for updates

(C) Author(s) (or their employer(s)) 2019. No commercial re-use. See rights and permissions. Published by BMJ.

To cite: Harun SN,
Holford NHG, Grimwood K,
et al. Thorax
2019;74:740-748.

\section{ABSTRACT}

Background While Aspergillus detection rates in adults, adolescents and older children with cystic fibrosis (CF) have increased, the risk of acquiring this fungal pathogen in young children is unknown.

Aim To determine the risk and explanatory factors of acquiring Aspergillus in children with CF by age 5 years. Methods Cross-sectional analysis of clinical, bronchoalveolar lavage and treatment data from the Australasian Cystic Fibrosis Bronchoalveolar Lavage study was used to identify predictive factors for detecting Aspergillus at age 5 years. A parametric repeated time-to-event model quantitatively described the risk and factors associated with acquiring Aspergillus and Pseudomonas aeruginosa from birth until age 5 years. Results Cross-sectional analysis found that the number of $P$. aeruginosa eradication courses increased the odds of detecting Aspergillus at age 5 years (OR 1.61, 95\% CI 1.23 to 2.12). The median (IQR) age for the first $P$. aeruginosa positive culture was $2.38(1.32-3.79)$ years and 3.69 (1.68-4.74) years for the first Aspergillus positive culture. The risk of $P$. aeruginosa and Aspergillus events changes with time after the first year of study entry. It also decreases for $P$. aeruginosa after completing $P$. aeruginosa eradication (HR $0.15,95 \% \mathrm{Cl} 0.00$ to 0.79), but increases for Aspergillus events (HR 2.75, $95 \% \mathrm{Cl} 1.45$ to 5.41 ). The risk of acquiring both types of events increases after having had a previous event. Conclusion In young children with $C F$, completing $P$. aeruginosa eradication therapy and previous Aspergillus events are associated with increased risk of acquiring Aspergillus.

\section{INTRODUCTION}

Cystic fibrosis (CF) is an inherited, life-limiting chronic illness with most affected people dying in respiratory failure following persistent endobronchial infection, usually from Pseudomonas aeruginosa. ${ }^{1}$ Eradication of early $P$. aeruginosa infection before it becomes chronic and established is generally successful and this treatment is widely practised. ${ }^{2}$ However, despite these measures most patients eventually develop chronic $P$. aeruginosa infection leading to increased respiratory symptoms, reduced lung function and decreased survival. ${ }^{3-5}$ Chronic

P. aeruginosa infection is usually treated with long-term, inhaled antibiotic therapy. However,

\section{Key messages}

What is the key question?

- What are the explanatory factors for acquiring Aspergillus in children with cystic fibrosis (CF) aged $\leq 5$ years?

What is the bottom line?

- Intensive Pseudomonas aeruginosa eradication treatment involving 2 weeks of intravenous anti-pseudomonal antibiotics, followed by 8 weeks of tobramycin solution for inhalation is associated with an increased risk of acquiring Aspergillus in respiratory cultures from children with CF during the first 5 years of life.

Why read on?

- Recurrent events of Aspergillus and the period after eradication therapy for $P$. aeruginosa increased the risk of acquiring Aspergillus.

such treatment has been associated with airway dysbiosis and acquisition of other potential pathogens, including filamentous fungi from the Aspergillus genus. ${ }^{67}$

In CF, Aspergillus prevalence has increased recently, particularly involving Aspergillus fumigatus in the adolescent and adult age groups. ${ }^{8-10}$ Allergic bronchopulmonary aspergillosis ${ }^{11}$ and Aspergillus sensitisation ${ }^{12}$ are the most common clinical disorders associated with finding Aspergillus in CF patients and can be accompanied by deteriorating lung function. Aspergillus in children is also linked with an increased risk of pulmonary exacerbations requiring hospitalisation. ${ }^{13}$ Thus, identifying possible explanatory variables for acquiring Aspergillus is potentially important. Studies conducted in adults ${ }^{14}$ and older children ${ }^{15}$ identified age and long-term oral and inhaled antibiotics as important explanatory variables for acquiring Aspergillus. A recent study identified long-term therapy with inhaled antibiotics, oral macrolides and inhaled corticosteroids as significant risk factors for persistent Aspergillus in older children and adults with $\mathrm{CF},{ }^{16}$ however, the situation in younger children with $\mathrm{CF}$ aged $\leq 5$ years is unknown. ${ }^{17}$ 
While types of eradication therapy for new $P$. aeruginosa infections vary internationally between CF centres and with the clinical condition of the patient, most include short-term courses of inhaled antibiotics with or without intravenous anti-pseudomonal agents and/or oral ciprofloxacin. ${ }^{18-20}$ The relationship between Aspergillus and short-term courses of eradication therapy for $P$. aeruginosa infections in young children remains unknown. The Australasian Cystic Fibrosis Bronchoalveolar Lavage (ACFBAL) study, a randomised controlled trial, collected detailed longitudinal data, including airway microbiology and treatments used in young children with CF across the first 5 years of life and provided an opportunity to examine this issue. Consequently, we aimed to determine the predictive factors associated with Aspergillus positive bronchoalveolar lavage (BAL) cultures in children with CF at 5 years of age and over the first 5 years of life.

\section{METHODS}

\section{Subjects, study design and specimens}

This study was a secondary analysis of data collected as part of the ACFBAL study, which ran from 1999 to $2009 .{ }^{21}$ Briefly, infants detected by a new born screening programme and with features of classic CF (two or more of the following: two CF gene mutations, sweat chloride $>60 \mathrm{mmol} / \mathrm{L}$, pancreatic insufficiency or meconium ileus) were randomised to either BAL-directed therapy or standard care where clinical judgement and oropharyngeal (OP) swabs guided treatment of pulmonary exacerbations in the first 5 years of life. As part of their routine care, infants recruited in three of the eight participating centres also received daily oral flucloxacillin as anti-staphylococcal prophylaxis until their first birthday.

Pulmonary exacerbations were defined as any change in respiratory symptoms from baseline. When a child in either study arm had an exacerbation, an OP specimen was obtained, and oral, non-antipseudomonal antibiotics were commenced.

Children in the BAL-directed arm had a BAL at baseline (before age 6 months), at the study end (at age 5 years), and after completion of any $P$. aeruginosa eradication therapy. These BAL specimens were termed 'surveillance' samples. In the BAL-directed study arm, BAL was also undertaken for exacerbations not responding to oral antibiotics in the community and requiring admission to hospital for intravenous therapy or if an OP culture was positive for $P$. aeruginosa. These BAL specimens were labelled 'symptomatic' samples. At the end of the study, all participants from both BAL-directed and standard care arms underwent a routine BAL at age 5 years when they were clinically stable. $^{21}$

In both ACFBAL treatment arms, a confirmed $P$. aeruginosa infection (defined by $\geq 10^{3}$ colony-forming units (CFU)/mL of $P$. aeruginosa from BAL cultures in the BAL arm and from any growth of $P$. aeruginosa in OP cultures from children in the standard arm) was treated identically with a course of eradication therapy. ${ }^{21}$ Eradication therapy consisted of 2 weeks of intravenous tobramycin and either intravenous ticarcillin-clavulanate or ceftazidime, followed by 4 weeks of oral ciprofloxacin and 8 weeks of nebulised tobramycin solution for inhalation. Further BAL (BAL-directed arm) or OP (standard care arm) cultures were performed following completion of every eradication treatment course and the eradication protocol was repeated if $P$. aeruginosa persisted. Children who were unwell and had positive BAL cultures with low counts of $P$. aeruginosa (ie, $<10^{3}$ $\mathrm{CFU} / \mathrm{mL}$ ) received the routine course of treatment for an exacerbation that consisted of 2 weeks of intravenous tobramycin and either intravenous ticarcillin-clavulanate or ceftazidime, but without subsequent nebulised tobramycin solution for inhalation or oral ciprofloxacin. Apart from managing P. aeruginosa infections, hospitalised treatment was individualised by the local team and, in the absence of positive $P$. aeruginosa cultures, this might include other non-antipseudomonal antibiotics.

Since no diagnostic threshold for infection with Aspergillus in BAL cultures has been determined and as its pathogenetic role is yet to be fully established, ${ }^{22}$ for this study we adopted a conservative approach and accepted any growth of Aspergillus in BAL fluid as indicating acquisition without asserting this was infection. As described previously, BAL and OP specimens were plated onto selective and non-selective media for counting and identifying respiratory pathogens by standard microbiological techniques. $^{23}$ Diagnostic microbiology laboratories at five of the eight CF centres employed Sabouraud dextrose agar with gentamicin routinely as selective media to help isolate fungal organisms from BAL cultures (see online supplementary digital content).

\section{Analysis}

Two separate analyses were undertaken. The first analysis aimed at identifying explanatory factors associated with any growth of Aspergillus in BAL cultures at age 5 years using a cross-sectional analysis of data from the entire study cohort. The second was a longitudinal analysis using BAL culture data confined to the BAL-directed therapy group to determine both the risk of having positive Aspergillus cultures from birth until age 5 years and the explanatory factors influencing this risk over time.

\section{Cross-sectional analysis at age $\mathbf{5}$ years}

The cross-sectional analysis employed univariable and multivariable logistic regression to evaluate the association between explanatory factors and positive Aspergillus BAL culture events at age 5 years using NONMEM v.7.4.1, ${ }^{24}$ Perl speaks NONMEM (PsN) V.4.1.0. ${ }^{25}$ Model selection was based on the likelihood ratio test, parameter estimate CIs and biological plausibility. An explanatory variable was retained in the model, if it provided a better model fit, which was based on the goodness of fit criteria (change in objective function value $(\Delta \mathrm{OFV})$ ) with a significance level of $\mathrm{p}<0.05$ (corresponding to $\Delta \mathrm{OFV}>3.84$ ).

\section{Longitudinal analysis from enrolment at age $<6$ months until 5 years of age}

The longitudinal parametric survival analysis was performed using interval-censored repeated time-to-event (RTTE) models to determine the risk of acquiring recurrent positive Aspergillus BAL cultures in the first 5 years of life. The term $P$. aeruginosa event was defined by detection of $P$. aeruginosa (BAL culture $\geq 10^{3} \mathrm{CFU} / \mathrm{mL}$ ) and an Aspergillus event by any growth of Aspergillus in surveillance and symptomatic BAL cultures. To test the hypothesis generated from the cross-sectional analysis, a RTTE model for positive $\left(\geq 10^{3} \mathrm{CFU} / \mathrm{mL}\right)$ P. aeruginosa BAL cultures was built concurrently with an Aspergillus RTTE model. The joint model allows for the influence of the P. aeruginosa eradication therapy on the risk of having Aspergillus positive cultures to be evaluated. The actual event times for $P$. aeruginosa and Aspergillus events were not known except that they occurred at some time between the previous BAL and the current positive BAL procedure. All event times were treated as interval censored data, which means the event was assumed to occur at an unknown time during a known interval (see online supplementary digital content for details). The final joint model of repeated-time to Aspergillus and $P$. aeruginosa events was 
Table 1 Characteristics of subjects with positive and negative BAL cultures for Aspergillus at age 5 years, presented as number of subjects in each category (\%) or median (IQR) values

\begin{tabular}{|c|c|c|c|}
\hline Characteristics & $\begin{array}{l}\text { Positive Aspergillus } \\
\mathrm{n}=28(17.9 \%)\end{array}$ & $\begin{array}{l}\text { Negative Aspergillus } \\
\mathrm{n}=128(82.1 \%)\end{array}$ & $\begin{array}{l}P \\
\text { value }\end{array}$ \\
\hline Female & $17(60.7)$ & $60(46.9)$ & 0.23 \\
\hline Meconium ileus & $7(25.0)$ & $25(19.5)$ & 0.34 \\
\hline Children in BAL arm of ACFBAL study & $15(53.6)$ & $64(50.0)$ & 0.52 \\
\hline Children in OP arm of ACFBAL study & $13(46.4)$ & $64(50.0)$ & \\
\hline Received gentamicin & $8(28.6)$ & $35(27.3)$ & 0.53 \\
\hline Received anti-staphylococcal prophylaxis until their first birthday & $6(21.4)$ & $27(21.1)$ & 0.56 \\
\hline $\begin{array}{l}\text { Children with positive Pseudomonas aeruginosa in BAL culture at age } \\
5 \text { years }\end{array}$ & $4(14.3)$ & $13(10.2)$ & 0.36 \\
\hline Children with positive $P$. aeruginosa in BAL culture prior to age 5 years & 8 (28.6) & $37(28.9)$ & 0.59 \\
\hline Body mass index z-score at BAL & $\begin{array}{l}0.16 \\
(-0.31,0.58)\end{array}$ & $\begin{array}{l}0.01 \\
(-0.41,0.43)\end{array}$ & 0.45 \\
\hline Cumulative dosage of intravenous tobramycin (not associated with eradication therapy) (mg) & $\begin{array}{l}33 \\
(0.00,49.5)\end{array}$ & $\begin{array}{l}64 \\
(0.0,670.0)\end{array}$ & 0.03 \\
\hline Cumulative dosage of intravenous tobramycin received $(\mathrm{mg})^{*}$ & $\begin{array}{l}2667.5 \\
(247.5,4203.8)\end{array}$ & $\begin{array}{l}0.0 \\
(0.0,2344.5)\end{array}$ & 0.001 \\
\hline Cumulative dosage of inhaled tobramycin received $(\mathrm{mg})$ & $\begin{array}{l}56282.5 \\
(8449.5,72750)\end{array}$ & $\begin{array}{l}0.0 \\
(0.0,36600)\end{array}$ & 0.06 \\
\hline Cumulative dosage of intravenous and oral antibiotics (not associated with eradication therapy) (mg) $\dagger$ & $\begin{array}{l}11784.9 \\
(1458.7,43099.5)\end{array}$ & $\begin{array}{l}7323.5 \\
(468.75 \\
33186.5)\end{array}$ & 0.26 \\
\hline Minimum annual temperature at geographic region at baseline $\left({ }^{\circ} \mathrm{C}\right)$ & $\begin{array}{l}10.6 \\
(-4.53,11.8)\end{array}$ & $\begin{array}{l}11.9 \\
(4.4,12.8)\end{array}$ & 0.14 \\
\hline Maximum annual temperature at geographic region at baseline $\left({ }^{\circ} \mathrm{C}\right)$ & $\begin{array}{l}27.2 \\
(20.6,31.4)\end{array}$ & $\begin{array}{l}24.1 \\
(20.6,26.7)\end{array}$ & 0.045 \\
\hline Number of $P$. aeruginosa eradication therapy courses received before BAL & $2(1,2)$ & $0.5(0,1)$ & 0.001 \\
\hline Number of 'all-cause' CF-related hospitalisations before age 5 years BAL & $3.5(1,7)$ & $3(1.2,5.5)$ & 0.03 \\
\hline Number of all pulmonary exacerbations before age 5 years BAL & $12(6.5,17.5)$ & $13(10,17)$ & 0.66 \\
\hline Number of pulmonary exacerbations requiring hospitalisation before age 5 years BAL & $3(1,4)$ & $2(1,4)$ & 0.90 \\
\hline
\end{tabular}

${ }^{*}$ Combined with an anti-pseudomonal beta-lactam antibiotic.

†Antibiotics other than tobramycin and not associated with eradication therapy (eg, intravenous cefuroxime, oral macrolides, amoxicillin-clavulanate and cotrimoxazole).

ACFBAL, Australasian Cystic Fibrosis Bronchoalveolar Lavage study; BAL, bronchoalveolar lavage; CF, cystic fibrosis;OP, oropharyngeal; n, number of children contributing data.

evaluated with a non-parametric bootstrap to assess parameter imprecision and a Kaplan-Meier visual predictive check (VPC) to assess the predictive performance (see online supplementary digital content). ${ }^{26}$ The RTTE model was extrapolated to illustrate the time to Aspergillus events based on occurrence of subsequent treatment for $P$. aeruginosa over 8 years for different clinical scenarios. The age of 8 years was selected to illustrate the probability of an event beyond the time of the available data (see online supplementary digital content).

\section{RESULTS}

\section{Factors associated with any growth of Aspergillus in BAL} cultures at age 5 years: univariable and multivariable logistic regression analysis

Overall, 156 end-of-study BAL cultures from 156 children (49.4\% female) were obtained at a median age of 5.05 years (range 4.77-6.30). Twenty-eight (17.9\%) of these cultures grew Aspergillus, including 12/45 (26.7\%) BAL cultures from children who had received $P$. aeruginosa eradication therapy previously (table 1).

Associations between variables and positive Aspergillus BAL culture at age 5 years are represented in univariable and multivariable logistic regression as shown in table 2 and online supplementary table $1 \mathrm{E}$.

An independent association was found between BAL cultures for Aspergillus and number of eradication courses for $P$. aeruginosa received before the end-of-study BAL at age 5 years. Maximum temperature of the geographic location at birth, as a surrogate for local climate and environmental factors, improved the fit of the model at initial inclusion; however, was eliminated during the backwards elimination step (table 2). Bootstrap results showed that the median bootstrap parameter estimate corresponded well with the model parameter estimates from the original data. The number of eradication courses received was the only variable in the univariable analysis predictive of positive Aspergillus BAL cultures at age 5 years with an OR of 1.61 (95\% CI 1.23 to 2.12) for every individual course received before a positive Aspergillus BAL culture.

Factors influencing risk of having positive Aspergillus cultures from enrolment at age $<6$ months until age 5 years: longitudinal analysis

Characteristics of children in the BAL-directed therapy group $(\mathrm{n}=80)$ used in the longitudinal analysis are shown in table 3. 
Table 2 Univariable and multivariable logistic regression analysis of variables associated with positive Aspergillus BAL cultures at age 5 years

\begin{tabular}{|c|c|c|}
\hline Univariable logistic regression & $\Delta \mathrm{OFV}$ & OR $(95 \% \mathrm{Cl})$ \\
\hline \multicolumn{3}{|l|}{ Categorical variable } \\
\hline Female & -1.77 & 0.57 (0.06 to 1.48$)$ \\
\hline Meconium ileus & -0.41 & $1.37(0.48$ to 5.81$)$ \\
\hline Children in BAL arm of ACFBAL study & -0.88 & 1.07 (0.47 to 2.41$)$ \\
\hline Received gentamicin & -0.01 & $1.06(0.25$ to 3.13$)$ \\
\hline $\begin{array}{l}\text { Received anti-staphylococcal prophylaxis until their } \\
\text { first birthday }\end{array}$ & -2.53 & 0.48 (0.30 to 5.32$)$ \\
\hline \multicolumn{3}{|l|}{ Continuous variables } \\
\hline Body mass index z-score at BAL & -0.65 & 0.89 (0.44 to 1.59$)$ \\
\hline $\begin{array}{l}\text { Cumulative dosage of intravenous tobramycin } \\
\text { received }(\mathrm{mg})^{*}\end{array}$ & -9.19 & 1.08 (0.99 to 1.10$)$ \\
\hline $\begin{array}{l}\text { Cumulative dosage of inhaled tobramycin received } \\
(\mathrm{mg})^{*}\end{array}$ & -2.88 & 1.00 (0.99 to 1.01$)$ \\
\hline $\begin{array}{l}\text { Cumulative dosage of intravenous tobramycin (not } \\
\text { associated with eradication therapy) (mg) }\end{array}$ & -3.42 & $1.12(1.00$ to 1.12$)$ \\
\hline $\begin{array}{l}\text { Cumulative dosage of intravenous and oral } \\
\text { antibiotics (not associated with eradication therapy) } \\
\text { (mg) } \dagger\end{array}$ & -0.77 & $1.00(1.00,1.00)$ \\
\hline $\begin{array}{l}\text { Minimum annual temperature at geographic region } \\
\text { at baseline }\left({ }^{\circ} \mathrm{C}\right)\end{array}$ & -2.07 & 0.96 (0.83 to 0.99$)$ \\
\hline $\begin{array}{l}\text { Maximum annual temperature at geographic region } \\
\text { at baseline }\left({ }^{\circ} \mathrm{C}\right)\end{array}$ & -3.89 & 1.09 (1.01 to 1.17$)$ \\
\hline \multicolumn{3}{|l|}{ Discrete variables } \\
\hline $\begin{array}{l}\text { Number of Pseudomonas aeruginosa eradication } \\
\text { therapy courses received before BAL } \neq\end{array}$ & -14.55 & 1.61 (1.23 to 2.12$)$ \\
\hline $\begin{array}{l}\text { Number of 'all-cause' CF-related hospitalisations } \\
\text { before age } 5 \text { years BAL }\end{array}$ & -3.94 & $1.11(0.78$ to 1.24$)$ \\
\hline $\begin{array}{l}\text { Number of pulmonary exacerbations requiring } \\
\text { hospitalisation before age } \\
5 \text { years BAL }\end{array}$ & -0.06 & 1.00 (0.86 to 1.06$)$ \\
\hline $\begin{array}{l}\text { Number of all pulmonary exacerbations before age } \\
5 \text { years BAL }\end{array}$ & -0.66 & $0.92(0.9$ to 1.07$)$ \\
\hline \multicolumn{3}{|l|}{ Multivariable logistic regression } \\
\hline $\begin{array}{l}\text { Base model+number of } P \text {. aeruginosa eradication } \\
\text { therapy courses received before BAL positive } \\
\text { cultures for Aspergillus }\end{array}$ & $\Delta \mathrm{OFV}$ & OR $(95 \% \mathrm{Cl})$ \\
\hline $\begin{array}{l}\text { Cumulative dosage of intravenous tobramycin } \\
\text { received (mg) }\end{array}$ & $-0.43 \S$ & 1.02 (0.95 to 1.09$)$ \\
\hline $\begin{array}{l}\text { Number of 'all-cause' CF-related hospitalisations } \\
\text { before age } 5 \text { years BAL }\end{array}$ & $-0.03 \S$ & 1.01 (0.98 to 1.02$)$ \\
\hline $\begin{array}{l}\text { Cumulative dosage of intravenous tobramycin (not } \\
\text { associated with eradication therapy) (mg) }\end{array}$ & $-0.10 \S$ & 1.01 (1.00 to 1.01$)$ \\
\hline $\begin{array}{l}\text { Maximum annual temperature of geographic } \\
\text { location at baseline }\left({ }^{\circ} \mathrm{C}\right)\end{array}$ & $-4.56 \S$ & $1.11(1.01$ to 1.20$)$ १ \\
\hline \multicolumn{3}{|l|}{ Final model } \\
\hline $\begin{array}{l}\text { Base model+number of } P \text {. aeruginosa eradication } \\
\text { therapy courses received before age } 5 \text { years BAL }\end{array}$ & \multicolumn{2}{|c|}{$-14.551 .61(1.23 \text { to } 2.12)^{\star *}$} \\
\hline \multicolumn{3}{|c|}{$\begin{array}{l}\text { *Combined with an anti-pseudomonal beta-lactam antibiotic. } \\
\text { †Antibiotics other than tobramycin and not associated with eradication therapy (eg, intravenous } \\
\text { cefuroxime, oral macrolides, amoxicillin-clavulanate and co-trimoxazole). } \\
\text { ‡Model carried forward from the univariable step to the multivariable step. } \\
\text { §Based on (Base+number of } P \text {. aeruginosa eradication therapy courses received before BAL). } \\
\text { ๆExcluded from the final model during backward elimination step as } p>0.01 \text {. } \\
\text { **Bootstrap results ( } 95 \% \mathrm{CI}) \text {. } \\
\text { ACFBAL, Australasian Cystic Fibrosis Bronchoalveolar Lavage study; BAL, bronchoalveolar lavage; CF, } \\
\text { cystic fibrosis; } \triangle \mathrm{OFV} \text {, change in objective function value. }\end{array}$} \\
\hline
\end{tabular}

Online supplementary figure E1A shows the distribution of the 53 observed surveillance and symptomatic Aspergillus events times.

The symptomatic Aspergillus event distribution suggested a non-constant hazard. Similarly, online supplementary figure
Table 3 Characteristics of children from the BAL-directed therapy group $(n=80)$ used in the longitudinal analysis

\begin{tabular}{ll}
\hline Characteristics & $\begin{array}{l}\text { Median (IQR) } \\
\text { or number of } \\
\text { subjects in each } \\
\text { category }(\%)\end{array}$ \\
\hline Age at enrolment (years) & $0.26(0.19,0.36)$ \\
\hline Age at last observation (years) & $5.06(5.02,5.14)$ \\
\hline Female & $39(48.8)$ \\
\hline CFTR (homozygous Phe508del) & $57(71.3)$ \\
\hline Pancreatic insufficiency & $77(96.3)$
\end{tabular}

Pseudomonas aeruginosa events (BAL culture $>10^{3} \mathrm{CFU} / \mathrm{mL}$ ) throughout the 5 years Number of children without $P$. aeruginosa event $35(43.8)$

Number of children with $P$. aeruginosa events

$\begin{array}{lc}\text { First event } & 45(56.3) \\ \text { Second event } & 26(32.5) \\ \text { Third event } & 12(15.0) \\ \text { Fourth event } & 5(6.3) \\ \text { Fifth event } & 1(1.3)\end{array}$

Age at $P$. aeruginosa event (years)

\begin{tabular}{ll} 
Age at first event & $2.38(1.32,3.79)$ \\
\hline Age at second event & $3.17(2.67,4.79)$ \\
\hline Age at third event & $3.97(3.68,4.75)$ \\
\hline Age at fourth event & $4.82(4.16,4.59)$ \\
\hline Age at fifth event & 5.18
\end{tabular}

Number of children receiving $P$. aeruginosa eradication therapy throughout the 5 years

\begin{tabular}{lr} 
One eradication therapy course & $19(23.8)$ \\
Two eradication therapy courses & $14(17.5)$ \\
Three eradication therapy courses & $7(8.8)$ \\
\hline Four eradication therapy courses & $4(5.0)$ \\
Five eradication therapy courses & $1(1.3)$
\end{tabular}

Aspergillus species events (any growth in BAL culture) throughout the 5 years

$\begin{array}{lc}\text { A. fumigatus } & 35(43.8) \\ \text { A. nidulans } & 1(1.3) \\ \text { Number of children without Aspergillus events } & 44(55.0)\end{array}$

Number of children with Aspergillus events

\begin{tabular}{lc} 
First event & $36(45.0)$ \\
Second event & $13(16.3)$ \\
\hline Third event & $4(5.0)$ \\
Fourth event & $1(1.3)$
\end{tabular}

Age at Aspergillus event (years)

Age at first event

$3.69(1.68,4.74)$

Age at second event

Age at third event

$4.35(3.70,5.11)$

Age at fourth event

5.07

Number of children with an Aspergillus event who received

P. aeruginosa eradication therapy throughout the 5 years

$\begin{array}{lc}\text { Zero eradication therapy course } & 23(28.8) \\ \text { One eradication therapy course } & 5(6.3) \\ \text { Two eradication therapy courses } & 3(3.8) \\ \text { Three eradication therapy courses } & 1(1.3)\end{array}$

Continued 


\begin{tabular}{ll}
\hline Table 3 Continued & \\
\hline & $\begin{array}{l}\text { Median (IQR) } \\
\text { or number of } \\
\text { subjects in each } \\
\text { category (\%) }\end{array}$ \\
\hline Characteristics & $3(3.8)$ \\
\hline Four eradication therapy courses & $1(1.3)$ \\
\hline Five eradication therapy courses & \\
\hline $\begin{array}{l}\text { Number of children with Aspergillus species who received } \\
\text { antifungal (itraconazole) treatment throughout the } 5 \text { years }\end{array}$ & $13(16.3)$ \\
\hline Yes & $23(28.8)$ \\
\hline No
\end{tabular}

Number of children with Aspergillus events who received antistaphylococcal prophylaxis until their first birthday

$\begin{array}{ll}\text { Yes } & 17(21.3) \\ \text { No } & 19(23.8)\end{array}$

Number of children who received gentamicin during the first 5 years of life

$\begin{array}{ll}\text { Yes } & 24(30.0) \\ \text { No } & 56(70.0)\end{array}$

BAL, bronchoalveolar lavage; CFTR, cystic fibrosis transmembrane conductance regulator; $\mathrm{CFU}$, colony-forming units.

E1B shows the distribution of 89 observed surveillance and symptomatic $P$. aeruginosa event times. The distribution of the symptomatic $P$. aeruginosa events also suggested a non-constant hazard, which was confirmed by RTTE modelling. Of note, 23/36 (63.9\%) children who had an Aspergillus event in the first 5 years of life did not have a concomitant $P$. aeruginosa positive BAL culture. These children had a BAL after being hospitalised for either a CF-related respiratory illness or for the routine end-of-study BAL where Aspergillus, but not P. aeruginosa, was detected.

The risk of $P$. aeruginosa and Aspergillus events was described by changes associated with time and by time varying and non-time varying risk factors (see online supplementary table E2).

In the child's first year of entering the study, the risk of acquiring $P$. aeruginosa had a HR of 0.399 (95\% CI 0.181 to 0.599). After the first and second $P$. aeruginosa events, the risk of acquiring the subsequent $P$. aeruginosa infection increased with a HR of 138 (95\% CI 50.6 to 1236). The risk increased even more after the third event (HR of 1033, 95\% CI 335 to 15998). However, immediately after completing $P$. aeruginosa eradication therapy, the P. aeruginosa risk decreased with a HR of 0.15 (95\% CI 0.00 to 0.79 ) (table 4).

As predicted by a Gompertz hazard model, the risk of acquiring Aspergillus event was very low during the first year of the study period (table 4). However, the risk then increased influenced by factors other than time alone. Having had the first Aspergillus event increased the risk of a second or third Aspergillus event as shown by HRs of $7.29 \times 10^{5}\left(95 \%\right.$ CI $1.99 \times 10^{5}$ to $\left.1.83 \times 10^{6}\right)$ and $5.97 \times 10^{5}\left(95 \%\right.$ CI $1.21 \times 10^{5}$ to $\left.2.05 \times 10^{6}\right)$, respectively. After completing $P$. aeruginosa eradication therapy, the Aspergillus risk increased with a HR of 2.75 (95\% CI 1.45 to 5.41).

Kaplan-Meier VPCs for $P$. aeruginosa events show good predictions (figure 1). Aspergillus events shown in figure 2 indicate that the final model describes the observed data generally adequately. Some discrepancies after 4 years of age were noted, showing a deficiency of the model to describe $P$. aeruginosa and Aspergillus events around that age (online supplementary figures E2 and E3). The evaluated RTTE model of Aspergillus events was then extrapolated to illustrate the probability of having recurrent Aspergillus events beyond the studied time, to 8 years of age, so as to demonstrate the results of the model regarding Aspergillus events in the near future for young children with $\mathrm{CF}$ under different hypothetical clinical scenarios (online supplementary figure E4).

\section{DISCUSSION}

Our study shows that 28/156 (17.9\%) children with CF participating in a randomised controlled trial of BAL-directed therapy versus standard management of acute pulmonary exacerbations had positive cultures for Aspergillus when all trial participants underwent a routine end-of-study BAL at 5 years of age. In the subgroup of children randomised to the BAL arm of the study, $36 / 80(45 \%)$ had at least one positive BAL culture for Aspergillus during their first 5 years of life.

In the current study, $27 \%$ of all children who had received antibiotics previously to eradicate $P$. aeruginosa had positive BAL cultures for Aspergillus at the time of their BAL at 5 years of age. This result is higher than the $11 \%{ }^{7}$ and $22 \%{ }^{6}$ reported for older children receiving long-term antibiotics for chronic $P$. aeruginosa infection. This may be related to sample type because BAL cultures might be more sensitive than sputum for detecting Aspergillus. ${ }^{27}$

$P$. aeruginosa infections were discontinuous, not time-varying events, and due to the study design these events contained the same information as eradication therapy. We jointly described the hazard for repeated $P$. aeruginosa based on the $P$. aeruginosa infection events and the hazard model of repeated Aspergillus events, which described the continuous probability of having one of these events over time. The risk of detecting Aspergillus was increased after completing $P$. aeruginosa eradication therapy rather than before (the difference between our model and the constant hazard model is described in online supplementary figure E5 of the online supplementary digital content). This suggests that either eliminating or suppressing $P$. aeruginosa may be the key reason for acquiring Aspergillus rather than the recent suggestion of each promoting the other's growth. ${ }^{28}$ This is biologically plausible since in-vitro studies found $P$. aeruginosa secreted phenazines that inhibited Aspergillus growth and biofilm formation by generating reactive oxygen and nitrogen species. $^{2930}$

$P$. aeruginosa eradication therapy is associated with reduced prevalence of chronic infection with this organism. ${ }^{82031}$ The type of eradication therapy varies in different settings from 1 month of either inhaled tobramycin alone ${ }^{13}$ or combined with oral ciprofloxacin, ${ }^{32}$ through to 3 months of combination oral and inhaled therapy, ${ }^{33}$ to regimens that include intravenous antibiotics. $^{21}$ The optimal eradication therapy is unknown ${ }^{20}$ and may vary by age, disease severity, adjunctive therapies and if it is an initial or recurrent new infection. ${ }^{34}$

In our study, we found that the baseline hazard for $P$. aeruginosa did not change greatly with time. The risk of $P$. aeruginosa events increased with the number of $P$. aeruginosa events and decreased after eradication therapy. This may suggest a potential lasting clinical benefit of eradication therapy for P. aeruginosa in young children with $\mathrm{CF}^{35}$

Eradication therapy in the ACFBAL study was for 10 weeks and included 8 weeks of inhaled tobramycin compared with the 4-week courses of inhaled tobramycin that are now recommended by some authorities. ${ }^{19}$ This longer course of inhaled antibiotic may have increased the risk of acquiring Aspergillus. We were unable to detect an increased risk of acquiring Aspergillus associated with 2 weeks of intravenous antibiotics alone 


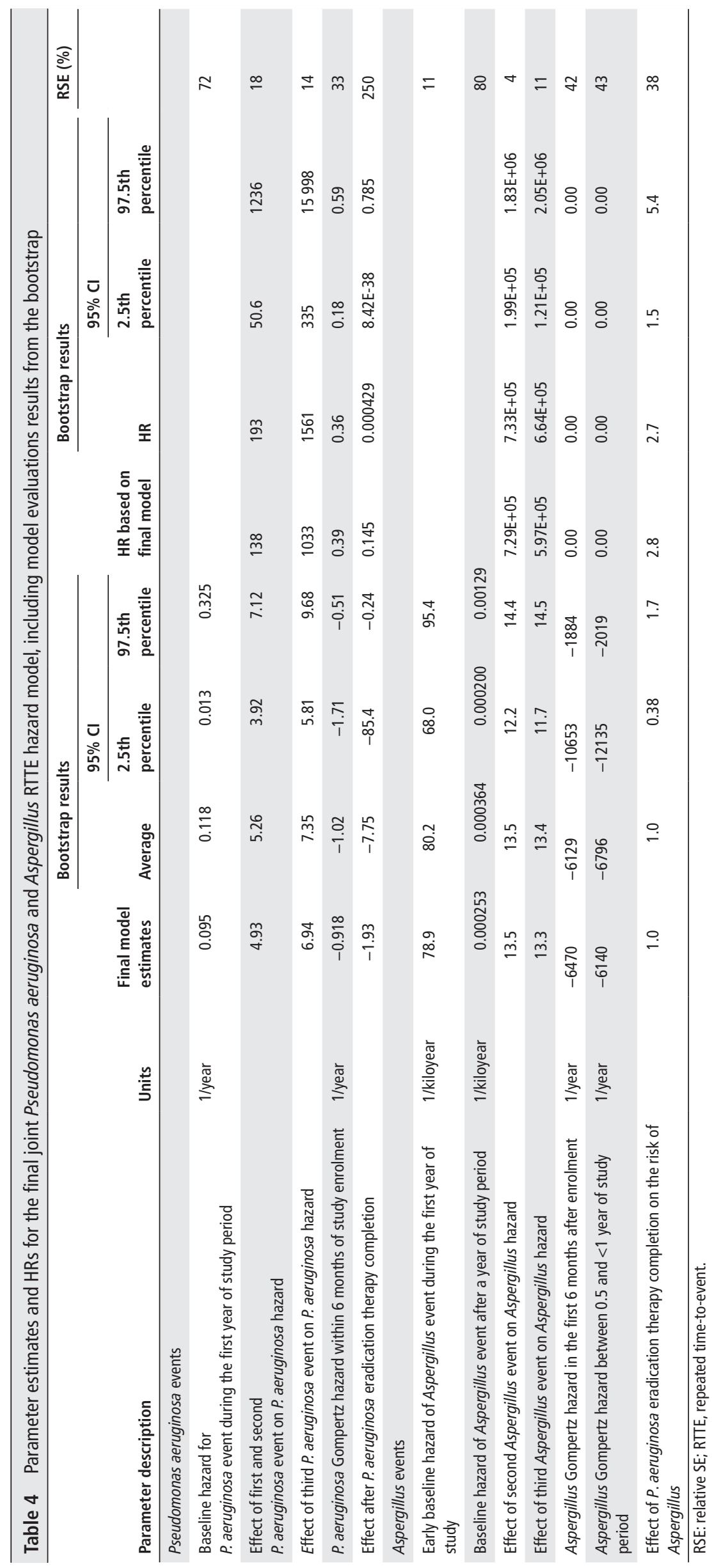

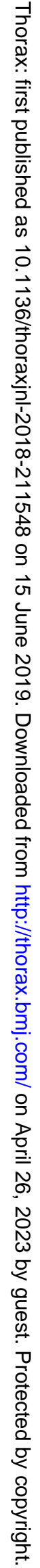




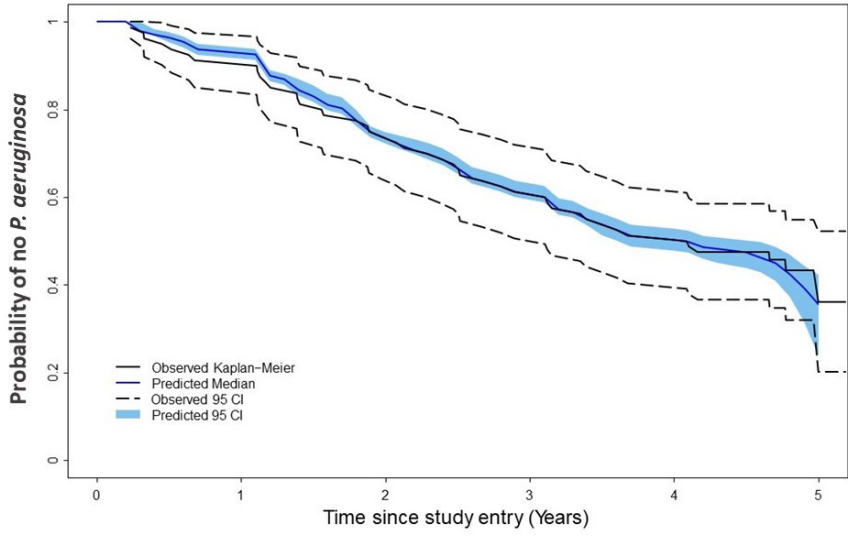

Figure 1 Kaplan-Meier plots showing the Pseudomonas aeruginosa survivor function (probability of no $P$. aeruginosa detection) throughout the first 5 years of life for the first four $P$. aeruginosa events. The observed data survivor function is described by a solid black line with 95\% Cls (dashed black lines). These are overlaid with the median predicted survivor function (solid blue line) and $95 \% \mathrm{Cl}$ (shaded area).

without inhaled tobramycin, which suggests that inhaled therapy may pose the greater risk. Clearly, we are unable to generalise the findings from this study to all forms of eradication therapy and eradicating $P$. aeruginosa remains an integral part of clinical care. Furthermore, admission to hospital was required for treating $P$. aeruginosa infections throughout the ACFBAL study, and exacerbations, $P$. aeruginosa and eradication therapy are necessarily inseparable in our analysis.

Studies are now needed to externally validate the evaluated hazard model. In the future, it will be of interest to determine whether antifungal therapy reduces the risk of Aspergillus events and if this confers a clinical benefit. However, this study was not designed to answer these questions and only 13 children with positive Aspergillus cultures received antifungal treatment.

CF genotype functional class has been reported previously as having an important effect on the age of first acquiring $P$. aeruginosa. ${ }^{36}$ However, the effects of CF transmembrane regulator mutations were not tested in the current study as 57/80 (71\%) children in the longitudinal analysis were homozygous for the

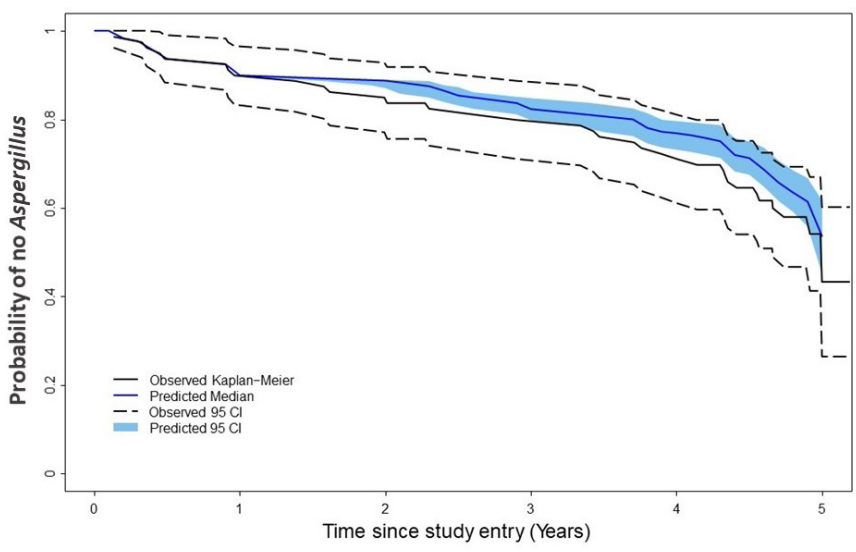

Figure 2 Kaplan-Meier plots showing the Aspergillus survivor function (probability of no Aspergillus detection) throughout the first 5 years of life for the first four Aspergillus events. The observed data survivor function is described by a solid black line with $95 \% \mathrm{Cls}$ (dashed black lines). These are overlaid with the median predicted survivor function (solid blue line) and 95\% Cl (shaded area).
Phe508del mutation, and all children had positive sweat chloride testing and classical CF clinical features.

Other limitations are that the ACFBAL study was not able to control for the number of cultures obtained per child. Children with $P$. aeruginosa infection who received more courses of eradication therapy also provided more BAL cultures, which may have increased the likelihood of identifying Aspergillus in these children. However, $P$. aeruginosa infection was not the only reason for children undergoing BAL. Children with more prolonged or severe pulmonary exacerbations that warranted admission to hospital also had BAL cultures where $P$. aeruginosa infection was not detected. Moreover, pulmonary exacerbations were not found to increase the risk of Aspergillus species being cultured in BAL. Although the data were collected prospectively between 1999 and 2009, our analysis was exploratory thus causal inferences are only suggestive. Also accepting any positive Aspergillus BAL cultures in the analysis may have resulted in misclassification of children with transient, low-level colonisation or upper airway contamination. ${ }^{37}$ Furthermore, BAL processing protocols for fungi were not standardised between diagnostic laboratories and none employed more sensitive molecular-based detection techniques. However, five of eight centres included selective media to isolate fungal organisms ${ }^{38}$ and no clustering was observed between centres.

The study's strengths include employing BAL to detect Aspergillus species in the lower airways and the detailed, prospectively collected longitudinal data available on exacerbations, treatments and infection status. Furthermore, while the logistic approach is limited because it assumes detections are determined by factors present at the time of BAL, RTTE uses the full-time course of factors before the detection and the duration of exposure to the factors of interest.

In conclusion, we found that the risk of recurrent Aspergillus events in children with CF was very low in the first year after enrolment into the study and increased after completing $P$. aeruginosa eradication therapy and once children had an Aspergillus event. Studies are now needed to determine whether Aspergillus is simply a marker of the lower airway dysbiosis associated with CF lung disease with minimal clinical consequences or a cause of progressive lung injury. As a first step, we showed recently in children from the ACFBAL cohort that there was no association between positive Aspergillus BAL cultures and either impaired lung function or with lung function decline between ages 5 and 14 years although there was an association with air trapping on chest CT scan. ${ }^{39}$

Acknowledgements The authors acknowledge the contribution of the Australian Centre of Pharmacometrics (ACP) and the University of Auckland with respect to the NONMEM software license and hardware. The authors would like to thank Joyce Cheney and Nicholas Gailer for their assistance in data extraction. The authors would like to thank Michiel Joost van Esdonk from The University of Queensland and Leiden Academic Centre for Drug Research, Leiden University for his advice on logistic regression analysis.

Collaborators The following investigators constitute the ACFBAL Study Investigators Group: Claire E Wainwright, Lady Cilento Children's Hospital, Brisbane and The University of Queensland, Brisbane; Keith Grimwood, Griffith University; Joyce Cheney, Lady Cilento Children's Hospital, Brisbane; Narelle George, Pathology Queensland; Colin F Robertson, Royal Children's Hospital, Melbourne; Rosemary Carzino, Murdoch Children's Research Institute, Melbourne; Marj Moodie, Deakin University, Melbourne; David S Armstrong, Monash Medical Centre, Melbourne; Peter J Cooper, The Children's Hospital at Westmead, Sydney; A (James) Martin, Womens \& Children's Hospital, Adelaide; Bruce Whitehead, John Hunter Children's Hospital, Newcastle; Catherine A Byrnes, Starship Children's Hospital and University of Auckland, Auckland; Harm A Tiddens, Erasmus MC, Sophia Children's Hospital, Rotterdam, The Netherlands; Nicholas Gailer, B Creative Industries; Katrina Jess, Lady Cilento Children's Hospital, Brisbane; Peta Yarrow, Lady Cilento Children's Hospital, Brisbane; Merilyn McArthur, The Children's Hospital at Westmead, Sydney; Sam 
Forbes, The Children's Hospital at Westmead, Sydney; Hiran Selvadurai, The Children's Hospital at Westmead, Sydney; John Massie, Royal Children's Hospital, Melbourne; Sarath Ranganathan, Royal Children's Hospital, Melbourne; Phil Robinson, Royal Children's Hospital, Melbourne; Jan Tate, Starship Children's Hospital, Auckland; Els Van Der Wiel, Erasmus MC, Sophia Children's Hospital, Rotterdam, the Netherlands.

Contributors All authors meet the criteria of authorship. SNH was responsible for collecting and analysing the data and drafting the manuscript. NHGH performed and reviewed the longitudinal analysis, reviewed and edited the manuscript. CEW developed the study concept, supported data collection, reviewed and edited the manuscript. KG provided microbiological advice, reviewed and edited the manuscript. SH participated in concept development, reviewed and supported the analyses, reviewed and edited the manuscript.

Funding The original ACFBAL study was supported by the National Health and Medical Research Council (grants 9937868 and 351541) and the Children's Hospital Foundation Queensland, Australia. The tobramycin inhalation solution and delivery system used throughout the ACFBAL study was supplied by Pathogenesis Corporation, Chiron Corporation, and Novartis Pharmaceuticals Inc. CW was supported by a project grant from the Children's Hospital Foundation Brisbane and a Queensland Health Research Fellowship. SH was supported by a fellowship from the Alexander von Humboldt Foundation, Germany during part of the study.

Competing interests CEW reports grants from Australian National Health and Medical Research Council and non-financial support in providing study drug from Pathogenesis, then Chiron, and then Novartis during the conduct of the ACFBAL study, reports income on a per patient basis derived from Pharmaceutical StudiesVertex Pharmaceuticals, Boehringer-Ingelheim \& Ablynx NV. Epidemiological Research Support from GlaxoSmithKline-Analysis of Bronchoalveolar lavage (BAL) fluid from children with respiratory disorders; 2010-2012 Research Grant from Novo Nordisk Pharmaceuticals P/L- CF-IDEA Study; 2012-2013 Other Reimbursement: Vertex Pharmaceuticals Inc.-Consultant on the Vertex Physician Pediatric CF Advisory Board and the Vertex Innovation Awards (VIA) Grants Committee Gilead Sciences-AZLI Advisory Board Honorarium 2010 Medscape-Consultation interview regarding CF Studies 2012 Vertex Pharmaceuticals 2013 San Francisco return flight and accommodation as Investigator in Lumacaftor study (104) Vertex Pharmaceuticals 2014 return flight and accommodation + honorarium as invited speaker at European CF Conference, Gothenburg, Sweden Vertex Pharmaceuticals (Australia) 2015 honorarium as speaker at a Vertex sponsored educational meeting series Vertex Pharmaceuticals 2015 Chicago return flight and accommodation as Investigator in Lumacaftor study (109) Vertex Pharmaceuticals (Australia) 2015 honorarium for attendance at Advisory Board meeting at Australasian CF Conference held in Sydney in August; Vertex Pharmaceuticals P/L honorarium to attend the Vertex Steering Committee Meetings re VX15-770-123 Study and to attend the Global Medical Advisory Board Meeting in March 2016. Novartis Pharmaceuticals 2013 return travel and accommodation to give a symposium at European CF Conference in Lisbon Novartis Pharmaceuticals Australia P/L 2013 honorarium to present symposium at Australasian CF Conference in Auckland Novartis Pharmaceuticals 2014 return flight and accommodation + honorarium as invited speaker at National Pediatric Congress in Lebanon Novartis Pharmaceuticals Australia P/L 2015 honorarium to present symposium at Australasian CF Conference in Sydney in August; The University of Miami honorarium for meeting attendance. Thorax honorarium for associate editor duties Q3/Q4 2015 and BMJ honorarium for consulting work in April 2016. Current Board Positions-Thoracic Society Australia and New Zealand and International Advisory Board Vertex Pharmaceuticals P/L. (N.B. Payments for all items listed above have been made into an institutional consultancy fund account). KG reports grants from Australian National Health and Medical Research Council and non-financial support from Pathogenesis, then Chiron, and then Novartis during the conduct of the ACFBAL study.

\section{Patient consent for publication Obtained.}

Ethics approval The details of the ACFBAL study have been published previously and it was approved by the ethics committees of each of the eight participating centres.

Provenance and peer review Not commissioned; externally peer reviewed.

Data availability statement The Australasian Cystic Fibrosis BronchoAlveolar Lavage research group - listed in the authorship - have access to the ACFBAL data. Any decisions regarding the use of the data or publications within the group and/or access to the data from outside the group have been discussed and agreed by a core panel designated by the ACFBAL researchers.

\section{REFERENCES}

1 Elborn JS. Cystic fibrosis. Lancet 2016;388:2519-31.

2 Döring G, Flume P, Heijerman $\mathrm{H}$, et al. Treatment of lung infection in patients with cystic fibrosis: current and future strategies. J Cyst Fibros 2012;11:461-79.

3 Nixon GM, Armstrong DS, Carzino R, et al. Clinical outcome after early Pseudomonas aeruginosa infection in cystic fibrosis. J Pediatr 2001;138:699-704.
4 Kerem E, Viviani L, Zolin A, et al. Factors associated with FEV1 decline in cystic fibrosis: analysis of the ECFS patient registry. Eur Respir J 2014;43:125-33.

5 Sanders DB, Li Z, Laxova A, et al. Risk factors for the progression of cystic fibrosis lung disease throughout childhood. Ann Am Thorac Soc 2014;11:63-72.

6 Burns JL, Van Dalfsen JM, Shawar RM, et al. Effect of chronic intermittent administration of inhaled tobramycin on respiratory microbial flora in patients with cystic fibrosis. J Infect Dis 1999;179:1190-6.

7 Hodson ME, Gallagher CG, Govan JRW. A randomised clinical trial of nebulised tobramycin or colistin in cystic fibrosis. Eur Respir J 2002;20:658-64.

8 Lillquist YP, Cho E, Davidson AGF. Economic effects of an eradication protocol for first appearance of Pseudomonas aeruginosa in cystic fibrosis patients: 1995 vs. 2009. J Cyst Fibros 2011;10:175-80.

9 The Canadian Cystic Fibrosis Registry 2012 Annual Report. The Canadian cystic fibrosis registry 2014

10 Felton IC, Simmonds NJ. Aspergillus and cystic fibrosis: old disease - new classifications. Curr Opin Pulm Med 2014:20:632-8.

11 Mastella G, Rainisio M, Harms HK, et al. Allergic bronchopulmonary aspergillosis in cystic fibrosis. a European epidemiological study. Epidemiologic Registry of cystic fibrosis. Eur Respir J 2000;16:464-71.

12 Fillaux J, Brémont F, Murris M, et al. Assessment of Aspergillus sensitization or persistent carriage as a factor in lung function impairment in cystic fibrosis patients. Scand J Infect Dis 2012;44:842-7.

13 Amin R, Dupuis A, Aaron SD, et al. The effect of chronic infection with Aspergillus fumigatus on lung function and hospitalization in patients with cystic fibrosis. Chest 2010;137:171-6.

14 de Vrankrijker AMM, van der Ent CK, van Berkhout FT, et al. Aspergillus fumigatus colonization in cystic fibrosis: implications for lung function? Clin Microbiol Infect 2011;17:1381-6.

15 Jubin V, Ranque S, Stremler Le Bel N, et al. Risk factors for Aspergillus colonization and allergic bronchopulmonary aspergillosis in children with cystic fibrosis. Pediatr Pulmonol 2010;45:764-71.

16 Hong G, Psoter KJ, Jennings MT, et al. Risk factors for persistent Aspergillus respiratory isolation in cystic fibrosis. J Cyst Fibros 2018;17:624-30.

17 Surette MG. The cystic fibrosis lung microbiome. Ann Am Thorac Soc 2014;11 Suppl 1(Suppl 1):S61-S65.

18 Schelstraete P, Haerynck F, Van daele S, et al. Eradication therapy for Pseudomonas aeruginosa colonization episodes in cystic fibrosis patients not chronically colonized by $P$. aeruginosa. J Cyst Fibros 2013;12:1-8.

19 Mogayzel PJ, Naureckas ET, Robinson KA, et al. Cystic fibrosis Foundation pulmonary guideline. Pharmacologic approaches to prevention and eradication of initial Pseudomonas aeruginosa infection. Ann Am Thorac Soc 2014;11:1640-50.

20 Langton Hewer SC, Smyth AR. Antibiotic strategies for eradicating Pseudomonas aeruginosa in people with cystic fibrosis. Cochrane Database Syst Rev 2017;4.

21 Wainwright CE, Vidmar S, Armstrong DS, et al. Effect of bronchoalveolar lavagedirected therapy on Pseudomonas aeruginosa infection and structural lung injury in children with cystic fibrosis: a randomized trial. JAMA 2011:306:163-71.

22 Singh A, Ralhan A, Schwarz C, et al. Fungal pathogens in CF airways: leave or treat?. Mycopathologia 2018;183:119-37.

23 Armstrong DS, Grimwood K, Carlin JB, et al. Bronchoalveolar lavage or oropharyngeal cultures to identify lower respiratory pathogens in infants with cystic fibrosis. Pediatr Pulmonol 1996;21:267-75.

24 NONMEM User's Guides (1989-2009) [program]: Ellicott City, MD Icon development Solutions 2009

25 Lindbom L, Ribbing J, Jonsson EN. Perl-speaks-NONMEM (PsN)--a Perl module for NONMEM related programming. Comput Methods Programs Biomed 2004;75:85-94

26 Nguyen THT, Mouksassi M-S, Holford N, et al. Model evaluation of continuous data pharmacometric models: metrics and graphics. CPT Pharmacometrics Syst Pharmacol 2017:6:87-109.

27 Hogan DA, Willger SD, Dolben EL, et al. Analysis of lung microbiota in bronchoalveola lavage, protected brush and sputum samples from subjects with mild-to-moderate cystic fibrosis lung disease. PLoS One 2016;11:e0149998.

28 Zhao J, Cheng W, He X, et al. The co-colonization prevalence of Pseudomonas aeruginosa and Aspergillus fumigatus in cystic fibrosis: A systematic review and metaanalysis. Microb Pathog 2018;125:122-8.

29 Mowat E, Rajendran R, Williams C, et al. Pseudomonas aeruginosa and their small diffusible extracellular molecules inhibit Aspergillus fumigatus biofilm formation. FEMS Microbiol Lett 2010;313:96-102.

30 Kerr JR, Taylor GW, Rutman A, et al. Pseudomonas aeruginosa pyocyanin and 1-hydroxyphenazine inhibit fungal growth. J Clin Pathol 1999;52:385-7.

31 Stuart B, Lin JH, Mogayzel PJ. Early eradication of Pseudomonas aeruginosa in patients with cystic fibrosis. Paediatr Respir Rev 2010;11:177-84.

32 Treggiari MM, Retsch-Bogart G, Mayer-Hamblett N, et al. Comparative efficacy and safety of 4 randomized regimens to treat early Pseudomonas aeruginosa infection in children with cystic fibrosis. Arch Pediatr Adolesc Med 2011;165:847-56.

33 Høiby N, Frederiksen B, Pressler T. Eradication of early Pseudomonas aeruginosa infection. J Cyst Fibros 2005;4 Suppl 2:49-54. 


\section{Cystic fibrosis}

34 Blanchard AC, Horton E, Stanojevic S, et al. Effectiveness of a stepwise Pseudomonas aeruginosa eradication protocol in children with cystic fibrosis. J Cyst Fibros 2017; 16:395-400.

35 Langan KM, Kotsimbos T, Peleg AY. Managing Pseudomonas aeruginosa respiratory infections in cystic fibrosis. Curr Opin Infect Dis 2015;28:1-56.

36 Rosenfeld M, Emerson J, McNamara S, et al. Risk factors for age at initial Pseudomonas acquisition in the cystic fibrosis epic observational cohort. J Cyst Fibros 2012;11:446-53.
37 Rosenfeld M, Emerson J, Accurso F, et al. Diagnostic accuracy of oropharyngeal cultures in infants and young children with cystic fibrosis. Pediatr Pulmonol 1999;28:321-8.

38 Hong G, Miller HB, Allgood S, et al. Use of selective fungal culture media increases rates of detection of fungi in the respiratory tract of cystic fibrosis patients. J Clin Microbiol 2017;55:1122-30.

39 Harun SN, Wainwright CE, Grimwood K, et al. Aspergillus and progression of lung disease in children with cystic fibrosis. Thorax 2019;74. 